Check for updates

Cite this: RSC Adv., 2019, 9, 32753

Received 28th August 2019

Accepted 27th September 2019

DOI: 10.1039/c9ra06819h

rsc.li/rsc-advances

\title{
Enrichment of highly pure large-diameter semiconducting SWCNTs by polyfluorene- containing pyrimidine ring ${ }^{\dagger}$
}

\author{
Xia Wei and Xieraili Maimaitiyiming (D) *
}

\begin{abstract}
The use of copolymers to extract and purify semiconducting SWCNTS (sc-SWCNTs) and metallic SWCNTS ( $\mathrm{m}$-SWCNTs) is widely employed. In this paper, the performances of two pyrimidine-alt-dioctylfluorene conjugated polymers in the enrichment of semiconducting SWCNTs are compared, and the subtle structural effects on them are discussed. It was found that both pyrimidine-polymers were more effective in wrapping the semiconducting SWCNTs than the metallic SWCNTs under the co-action of the pyrimidine and fluorene rings. Moreover, methyl groups on the pyrimidine ring of the polymer slightly contributed to the semiconducting purity, and the minor differences of sc-SWCNTs extraction between two pyrimidine-polymers are compared. Additionally, the average diameter of the selected SWCNTs is larger than that of the raw SWCNTs. The experimental results show the excellent selectivity for SCSWCNT from both co-polymers: the index $\Phi_{\mathrm{i}}$ values for determining the purity of sC-SWCNTs were increased from 0.408 for P2 to 0.465 for P1, of which the selected sc-SWCNT purity is up to $99.9 \%$. The resulting purity and the inexpensive availability of pyrimidine co-polymers make them suitable to be considered for effective sc-SWCNT enrichment.
\end{abstract}

\section{Introduction}

Single-walled carbon nanotubes (SWCNTs), which can be considered as one-dimensional hollow tubular structures crimped by a single layer of graphene, have metallic or semiconducting properties associated with the diameter and chiral angle represented by a characteristic index $(n, m) \cdot{ }^{1-3}$ The carbon atoms in carbon nanotubes are dominated by $\mathrm{sp}^{2}$ hybridization. The curvature of the hexagonal lattice structure of carbon nanotubes forms a spatial topology, in which there are partial $\mathrm{sp}^{2}$ and $\mathrm{sp}^{3}$ mixing hybridizations in their structure defects. These $\pi$-orbitals overlap with each other to form a large, highly delocalized $\pi$-bond outside the carbon nanotube graphene sheets. It is the large $\pi$-bond on the outer surface of the carbon nanotube that provides a chemical basis for the combination of macromolecules with carbon nanotubes by non-covalent bonds.

Semiconducting carbon nanotubes (sc-SWCNTs) are the ideal channel materials for building super-performance fieldeffect transistors, chemical sensors and micro-logic circuits for high-precision electronics due to their high carrier mobility,

Key Laboratory of Energy Materials Chemistry, Ministry of Education, Key Laboratory of Advanced Functional Materials, Autonomous Region, Institute of Applied Chemistry, Key Laboratory of Oil and Gas Fine Chemicals, Educational Ministry of China, School of Chemistry and Chemical Engineering, Xinjiang University, Urumqi, 830046 Xinjiang, P. R. China.E-mail: 380731363@qq.com

$\dagger$ Electronic supplementary information (ESI) available. See DOI: 10.1039/c9ra06819h large current-to-switch ratio, and good stability. ${ }^{4-6}$ Therefore, obtaining high-purity sc-SWCNTs is of great significance for applications in nanoelectronic devices and other fields. The controllable achievement of high-quality sc-SWCNTs is consistently a focal research point these days. In the current research, high-purity sc-SWCNTs are often obtained by two methods, namely, the direct controllable preparation of high-quality scSWCNTs and the extraction of sc-WCNTs from commercial SWCNTs. ${ }^{7,8}$ Due to the complicated process and high cost of the former method, the latter was chosen to selectively enrich scSWCNTs using conjugated polymers on the basis of previous works. ${ }^{9-11}$ This method is not only easy to operate, but also controllable depending on the designable polymer.

Previous studies have shown that conjugated polymers, such as fluorene and fluorene derivatives, have good selectivity for large-diameter carbon nanotubes. ${ }^{\mathbf{1 2 - 1 4}}$ In recent decades, Adronov et al. reported that a large number of scientific studies have been proposed to investigate the performance of fluorene derivatives for the separation and enrichment of sc-SWCNTs and m-SWCNTs. ${ }^{15-18}$

Due to its large electronic delocalization system, fluorene was found to combine with carbon nanotubes by $\pi-\pi$ stacking. ${ }^{18-21}$ Researchers have been dedicated to studying a set of rules to further explain the mechanism of interaction between polymers and SWCNTs. Various kinds of conjugated polymers were synthesized, and different extraction behaviors were discussed with different monomers. ${ }^{22}$ 
Some studies on fluorene-alt-pyridine copolymer (synthesized by pulsed laser vaporization) have been reported to enrich large diameter sc-SWCNTs. ${ }^{23-26}$ In addition, the fluorene-altpyridine-3,5-diyl reported in the literature ${ }^{27,28}$ showed dispersibility, rather than selectivity, for SWCNTs in toluene solution. This can be attributed to the fact that the nitrogen atom of the nitrogen-containing aromatic heterocycle owns a pair of lone electrons, compared to the aromatic ring having $\mathrm{p}$ electron $\pi$ bond, and thus the affinity of the nitrogen-containing aromatic heterocycle to SWCNTs exhibits greater than that of fluorene to some extent. Therefore, it is meaningful to explore the pyrimidine ring with more than one $\mathrm{N}$ atom to selectively enrich sc-SWCNTs, in which relative wide energy gaps exist in comparison to m-SWCNTs.

In our work, fluorene-alt-pyrimidine-4,6-diyl conjugated polymers synthesized using a fluorene monomer and pyrimidine through the Suzuki coupling reaction were introduced, and their selectivity to plasma SWCNTs was investigated. The polymer/SWCNT dispersions were obtained by a simple ultrasonic dispersion and centrifugation. Then, the products were characterized by UV-visible-near-infrared (UV-vis-NIR), resonant Raman spectroscopy and photoluminescence spectroscopy (PL) and the results were discussed. Compared with previous studies, it was found that the polymer, in which the pyridine ring was replaced by the pyrimidine ring, exhibited a higher selectivity for sc-SWCNTs. Furthermore, in comparing the ability of the polymers for wrapping the SWCNTs, the presence of methyl groups on the 2-position of the pyrimidine ring of the polymer was found to selectively enrich sc-SWCNTs with high purity by more than $99.9 \%$. By analysing the SWCNT enrichment with the two polymers presented in this work and comparing with previous works, a proposition is presented for a future molecular design to separate high-purity sc-SWCNTs.

\section{Experimental}

\section{Materials}

All the chemicals used were of analytical grade. Dichloromethane, tetrahydrofuran (THF), toluene (TL) and palladiumtetrakis(triphenylphosphine) $\left(\mathrm{Pd}\left(\mathrm{PPh}_{3}\right)_{4}\right)$ were obtained from Shanghai Fine Chemical Materials Research Institute., Shanghai, China. 2,7-Bis(4,4,5,5-tetramethyl-1,3,2dioxaborolane-2-yl)-9,9-dioctylfluorene, $\mathrm{K}_{3} \mathrm{PO}_{4}$, hydrazine, 4,6dichloro-2-methylpyrimidine, and sodium dodecylbenzenesulfonate (SDBS) were purchased from J\&K Scientific Co. Ltd., Beijing, China and used as received. Purified plasma nanotube powder RN-220, was purchased from XFNANO Materials Tech Co, Ltd and used without any further treatment.

\section{Characterization}

Ultrasonication was done in a KQ5200B bath sonicator (KUN SHAN JIANG SU, China). UV-vis-NIR spectra were recorded using a Shimadzu UV-3600 spectrophotometer (Kyoto, Japan). Raman measurements were collected using a SENTERRA Raman spectrometer. A polytetrafluoroethylene (PTFE) filter (pore size, $0.2 \mu \mathrm{m}$, Millipore) was used for the filtration.
Photoluminescence spectroscopy measurements (PL) were collected using a FLS1000 Photoluminescence Spectrometer. Calculations were performed using the density functional theory (DFT) geometry optimizations of P1, P2 and P3 through Gaussian, at the level of RB3LYP using 6-311G (d, p) basis set, where the solvent was set to toluene.

\section{Enrichment of sc-SWCNTs}

$1.5 \mathrm{mg}$ of the as-synthesized polymer $\mathrm{P} 1$ or $\mathrm{P} 2^{29,30}$ (see ESI $\dagger$ for details) was dissolved in $3 \mathrm{~mL}$ toluene (TL), filtered and added to $1 \mathrm{mg}$ plasma SWCNTs, and then sonicated for $2 \mathrm{~h}$ (temperature control $20 \pm 5{ }^{\circ} \mathrm{C}$ ) to obtain the complex mixture. After the mixture was centrifuged at $20000 \mathrm{rpm}$ (28 $487.8 \mathrm{~g})$ for $30 \mathrm{~min}$ at $10{ }^{\circ} \mathrm{C}$, the resulting supernatant was used as the P1/SWCNTs composite dispersion. The other composites at different proportions of polymer/SWCNTs were obtained using the same process as described above (Scheme 1).

\section{Preparation of polymer/SWCNTs composite film}

The suspension of the filtered P1/SWCNTs was washed three times with $20 \mathrm{~mL}$ TL and then five times with $30 \mathrm{~mL}$ THF to remove excess polymer, and the filter paper was a $0.2 \mu \mathrm{m}$ organic nylon filter paper. In the illustration of Fig. $3 \mathrm{~b}$ (inset), the product that remained is shown on the filter paper. The filters were ultrasonically dispersed in $2 \mathrm{~mL}$ dichloromethane solution for 30 seconds to disperse the SWCNTs, and then dripcoated onto the silicon wafer surface for the Raman measurement. The P1 polymer sample solution $\left(0.5 \mathrm{mg} \mathrm{mL}^{-1}\right)$ and the composite sample dispersion were separately dispensed onto a glass slide and dried for the fluorescence test.

\section{Results and discussion}

UV-vis-NIR absorption spectroscopy is one of the important means to characterize the type of SWCNTs. The optical performance of a nanotube is closely related to its particular structure. Carbon nanotubes with different conductivities and diameters correspond to different characteristic absorption peaks, and the absorption band mainly originates from the optical transitions of the Van Hove singularities symmetrically distributed on both sides of the Fermi level. The electronic transition state between the valence band (v) and the conduction band (c) is reflected at
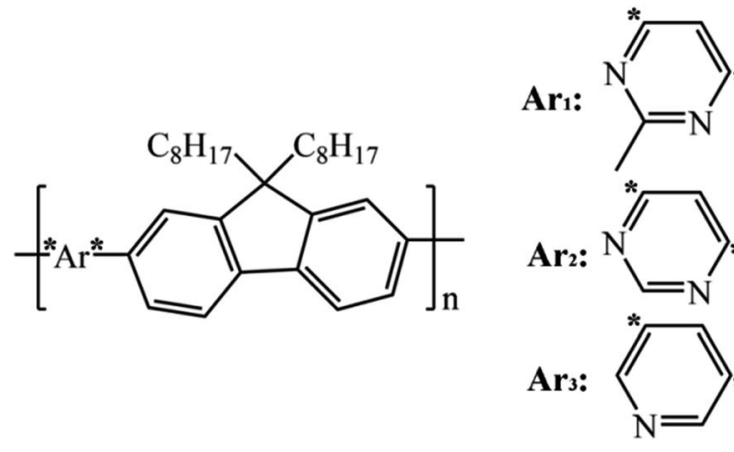

(P1)

Scheme 1 Molecular structure of P1, P2 and P3. 
different wavelengths in the absorption spectrum, and the concentration of different types of SWCNTs dispersed in the solvent can be determined by the absorption intensity. ${ }^{31}$

The SWCNTs were separately extracted and enriched by the two polymers with different substituents at a ratio of $1.5: 1$, and the results of the UV absorption spectra are compared in Fig. 1a. Compared to P2, a relatively deep gully appeared in the P1 (black) spectrum at 600-750 $\mathrm{nm}$ (assigned to the v1 $\rightarrow \mathrm{c} 1$ transition of m-SWCNTs), which indicates the presence of a purer semiconducting SWCNT type. In addition, there were identical peak profiles within the range of 750-1200 nm. The absorption peaks of the SWCNT dispersions extracted by P1 were expressed more clearly than those of the P2-extracted SWCNTs (red). This indicated the thorough dispersion of raw SWCNTs by both P1 and P2, but a slightly poorer selectivity of P2, which can be mainly explained by the strong Van der waals force between the raw SWCNTs and P2 molecules in the P2extracted system. In addition, this result was derived from the former's more facile v2 $\rightarrow$ c2 transition (S22) of sc-SWCNTs, preliminarily indicating the higher selectivity of $\mathrm{P} 1$ to scSWCNT. Compared to the literature, ${ }^{27}$ both pyrimidinepolymers in our work exhibited more appreciable selectivity than the pyridine-polymers. This may be due to the fact that compared to the pyridine ring, the increased number of $\mathrm{N}$ atoms on the pyrimidine ring induces the enhancement of the total number of free electrons, making it more inclined to select sc-instead of m-SWCNTs. For P1, its slightly higher selectivity for sc-SWCNTs can be explained by the overlap between one of the $\mathrm{sp}^{3}$ orbitals of the methyl group and the $\pi$-orbital on the pyrimidine unit in a phase-consistent direction. ${ }^{32,33}$ Due to the weak electron-donating ability of the methyl group, the electrons are uniformly delocalized throughout the pyrimidine ring, making the entire system more rich in electrons. Furthermore, the introduction of methyl groups for P1 increases the steric hindrance and thus, the conformation stability of the P1 molecules. ${ }^{13}$ The total electron density calculation mapped with the electrostatic potentials (ESP) and the total energy of the three polymers are shown in Fig. 1b. Both P1 and P2 exhibit lower energy values and are more stable than P3, thereby decreasing the dispersion and increasing the selectivity.

In addition, the reported method for estimating the relative purity of sc-SWCNTs can be approximated to evaluate the purity
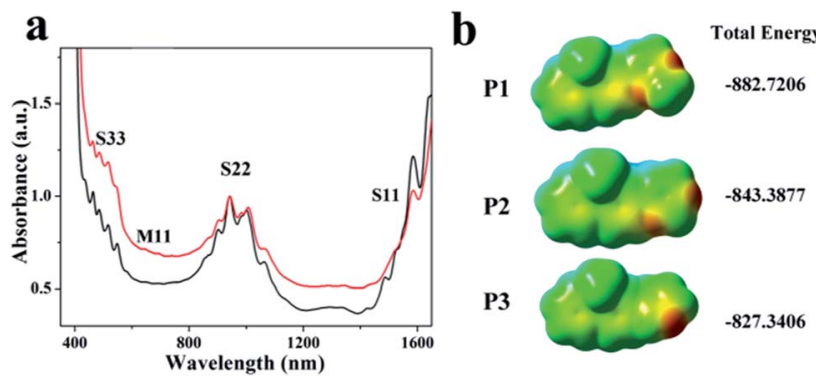

Fig. 1 (a) The UV absorption spectra of P1- (black) and P2-based (red) SWCNT dispersions. (b) The total electron density calculation of P1, P2 and P3. of the P1-enriched sc-SWCNTs. ${ }^{13,20}$ The $\Phi_{\mathrm{i}}$ value is defined as the ratio of the peak area to the background area in the range of S22 and M11 in the absorption spectrum, which can be used to evaluate the purity of sc-SWCNTs in the sample. A $\Phi_{\mathrm{i}}$ value that is greater than 0.4 corresponds to an excess of $99.9 \%$ of the scSWCNTs in the sample. ${ }^{\mathbf{1 0}, 14}$ The $\Phi_{\mathrm{i}}$ values of the P1 and P2enriched sc-SWCNTs at a ratio of $1.5: 1$ correspond to 0.243 and 0.156 , respectively (depicted in Fig. $\mathrm{S} 2 \dagger$ ).

The same polymer/SWCNT composites were measured at three excitation wavelengths of $532 \mathrm{~nm}$ (Fig. S3†), $633 \mathrm{~nm}$ and $785 \mathrm{~nm}$ to obtain the Raman spectra. RBM is a vibrational mode in which all carbon atoms move along the radial direction, and is thereby closely related to the nanotube diameter. ${ }^{34-39}$ The Raman spectra were collected at the three excitation wavelengths for the P1/SWCNTs and P2/SWCNTs, both of which were combined in the same substrate ratio of $1.5: 1$. It can be clearly seen from the Raman spectrum in Fig. 2 that both the P1 and $\mathrm{P} 2$ polymers have a tendency of enriching the sc-SWCNTs from the raw SWCNTs. Although the P1- and P2-extracted products at a ratio of $1.5: 1$ both exhibited a small and broad peak at the range of $135-175 \mathrm{~cm}^{-1}$ (corresponding to -SWCNT) at $785 \mathrm{~nm}$ wavelength excitation, a more distinctly sharp peak appeared at $190-240 \mathrm{~cm}^{-1}$, corresponding to the characteristic peaks of sc-SWCNTs, which further confirmed the selectivity of $\mathrm{P} 1$ and $\mathrm{P} 2$ for sc-SWCNTs.

As depicted in Fig. 2b, the G band belongs to the stretching motion of the atom pair in the carbon tube; both spectra exhibited a $\mathrm{G}^{+}$peak at the $633 \mathrm{~nm}$ laser excitation wavelength. The $\mathrm{G}^{+}$band at $1590.81 \mathrm{~cm}^{-1}$ (1589.72 $\mathrm{cm}^{-1}$ for P2/SWCNTs) and the $\mathrm{G}^{-}$band at $1571.83 \mathrm{~cm}^{-1}$ of $\mathrm{P} 1 / \mathrm{SWCNTs}$ were all sharper and clearer than those of P2/SWCNTs. In addition, the $\mathrm{G}^{-}$band of P1/SWCNTs was less intense and at a lower frequency compared to P2/SWCNTs, which was characteristic of the sc-SWCNTs. This result can be explained by the fact that the $\mathrm{G}^{+}$band originated from the carbon atom vibrations of the scSWCNTs along the nanotube axial length orientation (LO), and the less intense and sharper $\mathrm{G}^{-}$band originated from the carbon atom vibrations of the sc-SWCNTs along the nanotube tangential orientation (TO) axis. $^{29,30}$

It can be said that two factors contributed to the ability of the two polymers to extract sc-SWCNTs: first, it is the $\pi-\pi$ stacking of the carbon nanotubes and the fluorene ring that play a main role in the extraction. Second, the existence of the pyrimidine
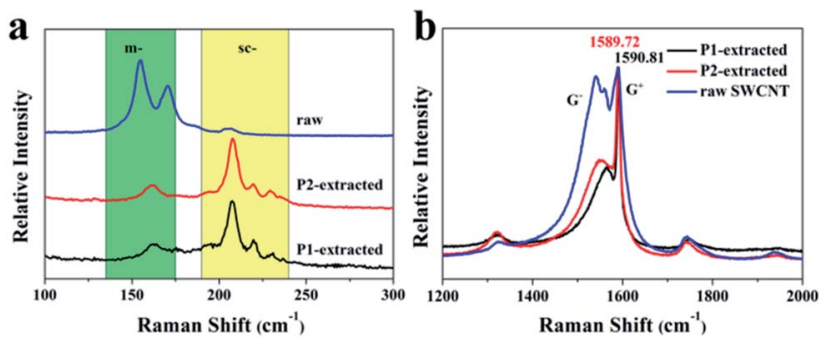

Fig. 2 The RBM range of the Raman spectra with laser excitation at $785 \mathrm{~nm}$ (a) and the G-band range at $633 \mathrm{~nm}$ laser excitation (b) corresponding to the P1-, P2-extracted and raw SWCNTs. 
ring negatively charges the polymer, and thus electrostatic attraction exists between the polymer and sc-SWCNTs. In addition, the presence of the methyl group attached onto the pyrimidine ring of the polymer (P1) increases the electron cloud density of the entire conjugated system, making it slightly easier for the $\pi-\pi$ stacking and electrostatic interaction with the scSWCNTs. Furthermore, the presence of the methyl group increases the dihedral angles of the pyrimidine and fluorene rings from $166.6^{\circ}$ of $\mathrm{P} 2$ to $167.1^{\circ}$ of $\mathrm{P} 1$ according to the calculation. This change possibly increases the ability of the entire molecular chain to effectively wrap around the larger sized scSWCNTs; thus, the $\pi-\pi$ interaction between P1 and the scSWCNTs is enhanced. The stability is further confirmed by the comparison of the HOMO and LUMO energy level difference between $\mathrm{P} 1(\Delta E=0.15353)$ and $\mathrm{P} 2(\Delta E=0.15201)$.

The absorption spectra of the $\mathrm{P} 1 / \mathrm{SWCNT}$ dispersion at different substrate ratios of P1:SWCNTs and the unsorted SWCNTs dispersed in SDBS solution are presented in Fig. 3a, and show the absence of the metallic part in the sorted section. The selectivity increases regularly with a decrease in the amount of P1. Fig. 3b shows the purity of the P1-selected SWCNTs displayed with a $\Phi_{\mathrm{i}}$ value of 0.438 at a substrate ratio of $0.5: 1$. In addition, the UV-vis-NIR spectrum of the P2/SWCNT dispersion is presented in Fig. S1 $\uparrow$ at different substrate ratios. The P2/ SWCNT dispersion exhibits increasing selectivity of scSWCNTs with decreasing substrate ratio, and the $\Phi_{\mathrm{i}}$ value reaches up to 0.408 at a substrate ratio of $0.5: 1$. It was demonstrated that both the P1- and P2-containing pyrimidine-4,6-diyls exhibited higher selectivity than pyridine-2,5-diyl. Fig. S4† shows the normalized absorption curves of the three sets of parallel samples of P1-extracted SWCNTs at a ratio of $0.5: 1$ at $942 \mathrm{~nm}$, thus confirming the repeatability of the P1 extraction. The absorption intensities ( $\Phi_{\mathrm{i}}$ value) corresponding to the $942 \mathrm{~nm}$ peak reach up to 0.055 (0.417), 0.149 (0.413), and 0.087
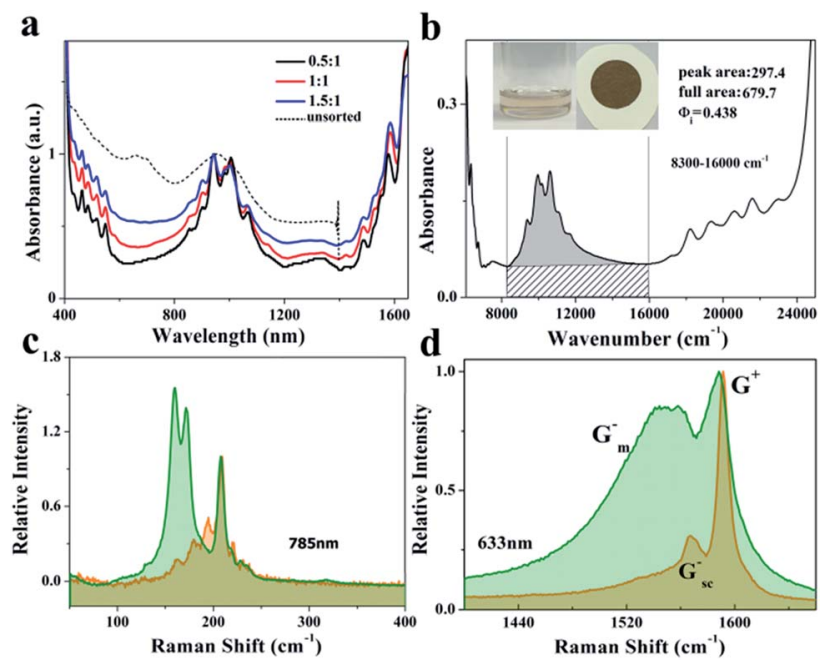

Fig. 3 (a) Absorption spectrum of P1/SWCNT dispersion at different ratios and of the unsorted SWCNT; the $\Phi_{\mathrm{i}}$ calculation of the P1extracted SWCNT $(0.5: 1)$ dispersion presented an integrated intensity of the S22 and M11 peaks from $8300 \mathrm{~cm}^{-1}$ to $16000 \mathrm{~cm}^{-1}$ (b), and the corresponding Raman spectra excited at $785 \mathrm{~nm}$ (c) and $633 \mathrm{~nm}$ (d).
(0.465). Furthermore, the purity level was compared between the as-obtained SWCNTs by P1 at a ratio of $0.5: 1$ and the scSWCNTs with high purity $(99.9 \%)$ from Nanointegris (Fig. S5 $†$ ), and a higher purity was found to be obtained in our work. The P1/SWCNTs at a substrate ratio of $0.5: 1$ (Fig. 4) was selected for further discussion. The strong absorption in the range of $420-580 \mathrm{~nm}$ and $750-1200 \mathrm{~nm}$ are due to the $\mathrm{v} 3 \rightarrow \mathrm{c} 3$ (S33) and v2 $\rightarrow$ c2 transitions (S22) of the sc-SWCNTs, and almost no v1 $\rightarrow \mathrm{c} 1$ transition of the m-SWCNTs at $600-700 \mathrm{~nm}$ can be observed (the background is mainly due to the nonnanotube carbon impurities).$^{20}$ This may be attributed to the fact that the aggregates of SWNCTs were fully dispersed in the solution containing polymer during ultrasonication.

The combination of SWCNTs with polymers weakened the $\pi-\pi$ interaction between SWCNTs. This, in turn, led to the promoted integration of SWCNTs and polymers. ${ }^{15}$ The results obtained from the UV absorption spectra above can be further understood by the G-band analysis of resonance Raman spectroscopy. The Raman spectra of the P1/SWCNT $(0.5: 1)$ film were also obtained with the $532 \mathrm{~nm}, 633 \mathrm{~nm}$ and $785 \mathrm{~nm}$ laser excitation wavelengths (Fig. 3b-d). The Raman spectra (Fig. 3c) of the P1/SWCNTs and unextracted raw plasma (dispersed in dichloromethane and evenly distributed on the silicon wafer by the drop-coating method) were obtained using laser excitation at $785 \mathrm{~nm}$. In the RBM, the yellow part represents P1/SWCNTs showing the characteristic peak of the sc-SWCNTs. Comparatively, the characteristic absorption region of the m-SWCNTs presented in the green portion of the raw SWCNTs shows the strong discrimination ability of P1 to sc-SWCNTs. ${ }^{11-15}$ In the resonance Raman spectra excited by the $633 \mathrm{~nm}$ laser (Fig. 3d), a strong contrast was observed with the raw plasma SWCNTs of the G peak. The P1/SWCNTs at $1591.45 \mathrm{~cm}^{-1}$ of the $\mathrm{G}^{+}$band was assigned to the $\mathrm{LO}$ axial vibrations of the $\mathrm{C}$ atoms on scSWCNTs. In raw plasma, the $\mathrm{G}^{-}$peaks of the m-SWCNTs were wider, while the $\mathrm{G}^{-}$peak of the sc-SWCNTs of P1/SWCNTs were sharper and less intense. The intensity ratios of the G/D bands

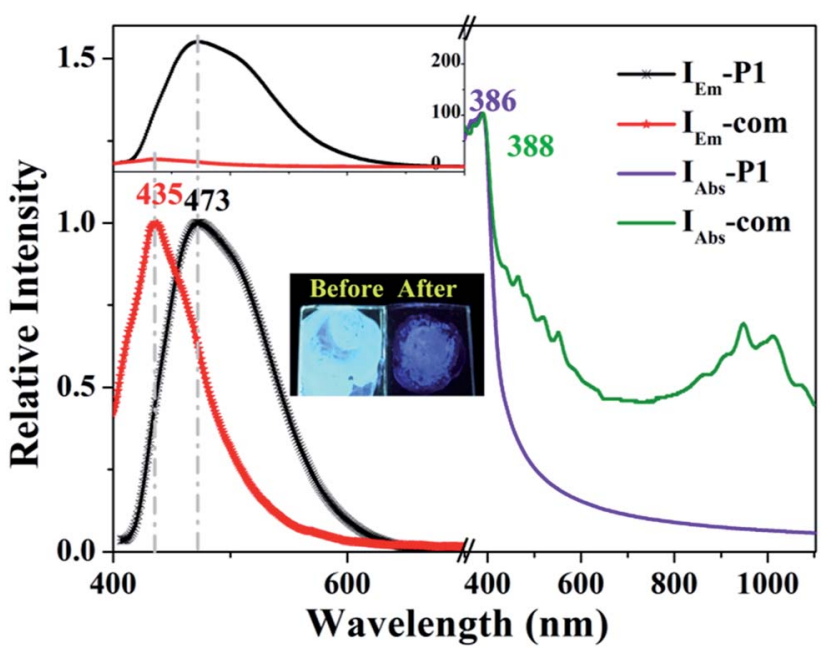

Fig. 4 Fluorescence emission intensity (left) and absorption spectra (right) of the sample films of polymer P1 and P1/SWCNTs compounds $(0.5: 1)$ (inset: the color contrast under the ultraviolet lamp). 
(peaks at $1591.45 \mathrm{~cm}^{-1}, 1309.23 \mathrm{~cm}^{-1}$, respectively) for $\mathrm{P} 1 /$ SWCNTs are $\sim 8.86$, implying that the SWCNTs treated with polymer sonication not only had high purity, but retained their structure well. Moreover, it has been proven that the RBM mode under different laser excitation wavelengths has a characteristic property: the diameter of the corresponding SWCNTs increases in a certain chirality index range with decreasing $\omega_{\mathrm{RBM}}{ }^{40-43}$ From Fig. S3, $\dagger$ it can be seen that the enriched sc-SWCNTs (red) exhibited a redshift with respect to the raw SWCNTs (black) with a difference of $11 \mathrm{~cm}^{-1}$. The peak width was relatively narrow, resulting in a larger average diameter compared with the raw SWCNTs.

The fluorescence emission intensity (left) and absorption spectrum (right) of both thin films of the polymer P1 and P1/ SWCNTs $(0.5: 1)$ composite are shown in Fig. 4 . The interaction of P1 with SWCNT enhanced the optical coupling between the ground state and the lowest excited state, passing through a significant blue shift with a fluorescence peak change from $473 \mathrm{~nm}$ of P1 $(2.62 \mathrm{eV})$ to $435 \mathrm{~nm}$ of P1/SWCNTs $(2.85 \mathrm{eV})$. The fluorescence of the composite was significantly weaker than that of the polymer itself under the illumination of the ultraviolet lamp, indicating that the presence of the SWCNTs caused significant photoquenching, which is consistent with the literature. ${ }^{4-46}$ The possible reason for this is that the energy difference existing between P1 and SWCNTs caused a photoinduced charge transfer at the interface between the two. ${ }^{\mathbf{4 7 - 5 1}}$ Subtle changes exist in the absorption spectra of the P1 film and its solution, including a more blunt peak than that of the P1 solution, which is the result of its interaction with the thin surface..$^{52,53}$

The PL measurement of SWCNTs dispersed in surfactants, and the electron absorption and emission transitions of more than 30 sc-SWCNTs have been revealed. However, because the metallic SWNTs have no band gap, fluorescence spectroscopy cannot specify the helical index of the metallic SWNTs. In addition, since large numbers of SWNT arrays, surface-grown SWNTs and nanotube bundles contain metallic SWNTs, the fluorescence of the semiconducting SWNTs is quenched, so fluorescence spectroscopy can only identify the spiral index of the single SWNT in suspensions and solutions. Therefore, the assignment $(n, m)$ of the sc-SWCNT is generally identified using a fluorescence spectrum. PL maps were recorded for the P1/ SWCNT dispersions $(0.5: 1)$ depicted in Fig. S6. $\dagger$ The locations of various SWCNT fluorescence maxima were assigned according to previously published data. ${ }^{\mathbf{4 9 0}}$ The intensities of scSWCNTs were observed, with most intense peak corresponding to the $(10,9),(12,4)$ and $(12,5)$ chiralities. In addition, it is obvious that redshifts occur in the range of 1500-1600 $\mathrm{nm}$ upon excitation. This may be attributed to the $\pi-\pi$ stacking with SWCNTs after being wrapped by $\mathrm{P} 1$, which affected the excitation of photons to SWCNTs.

\section{Conclusions}

In summary, we have compared and analyzed the performances of the two as-synthesized pyrimidine-fluorene co-polymers with previous works. Although the methyl-substituted pyrimidine brought subtle structural differences, the presence of the methyl group still brought a modest improvement to the scSWCNT extraction. We think it is the pyrimidine in the two pyrimidine-fluorene co-polymers that mainly contributed to the sc-SWCNT selectivity by increasing the electronic cloud density of their delocalization system. Furthermore, according to the calculation, the presence of the methyl group in the pyrimidinefluorene co-polymers increased the dihedral angles of the pyrimidine and fluorene rings from $166.6^{\circ}$ of $\mathrm{P} 2$ to $167.1^{\circ}$ of $\mathrm{P} 1$ and thus, the conformation stability of the P1 molecules (total energy: P1 -882.7206, P2 -843.3877). Therefore, compared to $\mathrm{P} 2$, P1 enriched mono-type sc-SWCNTs under the premise that the pyrimidine-alt-fluorene polymer exhibited high purity of selected sc-SWCNTs, which provided an important way to find sc-SWCNTs through a special pyrimidine structure. Nevertheless, a small amount of polymer-enriching SWCNTs still contained a few m-SWCNTs. Therefore, further studies on the determination of longer aliphatic chain substituents on pyrimidine rings, or the copolymerization of brominated fluorene monomer and methyl-pyrimidine in different proportions with the bis-(4,4,5,5-tetramethyl-1,3,2-dioxaborolane-2-yl)-9,9dioctylfluorene monomer may have more research implications for the extraction of higher-purity sc-SWCNTs.

\section{Conflicts of interest}

There are no conflicts to declare.

\section{Acknowledgements}

This work was supported by the National Natural Science Foundation of China (No. 51363020, 51763021, 21164012).

\section{Notes and references}

1 S. M. Bachilo, M. S. Strano, K. Carter, R. H. Hauge, R. E. Smalley and W. R Bruce, Science, 2002, 298, 2361.

2 D. Zhang, J. Yang, M. Li and Y. Li, ACS Nano, 2016, 10, 10789. 3 S. Augustine, J. Singh, M. Srivastava, M. Sharma and B. D. Malhotra, Biomater. Sci., 2017, 5, 901.

4 A. J. Kell, C. Paquet, O. Mozenson, I. Djavani-Tabrizi, B. Deore, X. Liu, G. P. Lopinski, R. James, K. Hettak and J. Shaker, ACS Appl. Mater. Interfaces, 2017, 9, 17226.

5 Y. Cao, S. Cong, X. Cao, F. Wu, Q. Liu, M. R. Amer and C. Zhou, Top. Curr. Chem., 2017, 375, 75.

6 S. Park, G. Pitner, G. Giri, J. H. Koo, J. Park, K. Kim, H. Wang, R. Sinclair, H.-S. P. Wong and Z. Bao, Adv. Mater., 2015, 27, 2656.

7 B. Liu, F. Wu, H. Gui, M. Zheng and C. Zhou, ACS Nano, 2017, 11, 31.

8 Y. Magnin, H. Amara, F. Ducastelle, A. Loiseau and C. Bichara, Science, 2018, 362, 212.

9 J. Vejpravova, B. Pacakova and M. Kalbac, Analyst, 2016, 141, 2639.

10 J. Lefebvre, J. Ding, Z. Li, P. Finnie, G. Lopinski and M. Prl, Acc. Chem. Res., 2017, 50, 2479. 
11 X. Mamtimin, R. Matsidik and I. Nurulla, Polymer, 2010, 51, 437.

12 S. Qiu, K. Wu, B. Gao, L. Li, H. Jin and Q. Li, Adv. Mater., 2019, 31, 1800750.

13 T. Lei, Y. C. Lai, G. Hong, H. Wang, P. Hayoz, R. T. Weitz, C. Chen, H. Dai and Z. Bao, Small, 2015, 11, 2946.

14 A. Chortos, I. Pochorovski, P. Lin, G. Pitner, X. Yan, T. Z. Gao, T. Jwf, T. Lei and H. P. Wong, ACS Nano, 2017, 11, 5660.

15 D. Fong, W. J. Bodnaryk, N. A. Rice, S. Saem, J. M. MoranMirabal and A. Adronov, Chem.-Eur. J., 2016, 22, 14409.

16 D. Fong, H. Zan, T. R. Wilks, R. K. O'Reilly and A. Adronov, J. Polym. Sci., Part A-1: Polym. Chem., 2017, 55, 2611.

17 D. Fong and A. Adronov, Chem. Sci., 2017, 8, 7292.

18 H. W. Lee, Y. Yoon, S. Park, J. H. Oh, S. Hong, L. S. Liyanage, H. Wang, S. Morishita, N. Patil and Y. J. Park, Nat. Commun., 2011, 2, 541.

19 W. Bodnaryk, N. Rice and A. Adronov, Polym. Chem., 2016, 7, 3832.

20 J. Ouyang, J. Ding, J. Lefebvre, Z. Li, C. Guo, A. J. Kell and M. Prl, ACS Nano, 2018, 12, 1910.

21 T. Lei, X. Chen, G. Pitner, H. S. P. Wong and Z. Bao, J. Am. Chem. Soc., 2016, 38, 802.

22 S. D. Stranks, S. N. Habisreutinger, B. Dirks and R. J. Nicholas, Adv. Mater., 2013, 25, 4365.

23 S. K. Samanta, M. Fritsch, U. Scherf, W. Gomulya, S. Z. Bisri and M. A. Loi, Acc. Chem. Res., 2014, 47, 2446.

24 A. Nish, J.-Y. Hwang, J. Doig and R. J. Nicholas, Nat. Nanotechnol., 2007, 2, 640.

25 K. S. Mistry, B. A. Larsen and J. L. Blackburn, ACS Nano, 2013, 7, 2231.

26 H. Wang and Z. Bao, Nano Today, 2016, 10, 737.

27 N. Berton, F. Lemasson, A. Poschlad, V. Meded, F. Tristram, W. Wenzel, F. Hennrich, M. M. Kappes and M. Mayor, Small, 2014, 10, 360 .

28 M. Tange, T. Okazaki and S. Iijima, ACS Appl. Mater. Interfaces, 2012, 4, 6458.

29 H. Fei, H. Lintao, W. Hongbin, W. Xiaohui, S. Huilin, C. Wei, Y. Wei and C. Yong, J. Am. Chem. Soc., 2004, 126, 9845.

30 H. Fei, H. Wu, D. Wang, W. Yang and C. Yong, Chem. Mater., 2004, 16, 708.

31 M. S. Strano, C. A. Dyke, M. L. Usrey, P. W. Barone, M. J. Allen, S. Hongwei, K. Carter, R. H. Hauge, J. M. Tour and R. E. Smalley, Science, 2003, 301, 1519.

32 H. Fujimoto and K. Fukui, Adv. Quantum Chem., 1972, 177, 6.
33 F. Mohri, K. Yoshizawa, T. Yambe, T. Ishida and T. Nogami, Mol. Eng., 1999, 8, 357.

34 S. V. Juan Ramon, D. Thomas, G. N. Oliver, S. Ivan, M. Andreas, J. Martin, A. Konstantin, R. Pascal and F. Roman, Nature, 2014, 512, 61.

35 C. Paola, S. Manuela, D. C. Maurizio, E. K. My Ali and R. Federico, Nanoscale, 2016, 12, 1611.

36 Y. Tian, H. Jiang, P. Laiho and E. I. Kauppinen, Anal. Chem., 2018, 90, 2517.

37 Y. Park, K. P. S. S. Hembram, R. Yoo, B. Jang, W. Lee, S.-G. Lee, J.-G. Kim, Y.-I. Kim, D. J. Moon and J.-K. Lee, J. Phys. Chem. C, 2019, 123, 14003.

38 M. Shim, T. Ozel, A. Gaur and C. Wang, J. Am. Chem. Soc., 2006, 128, 7522.

39 Y. Piao, J. R. Simpson, J. K. Streit, G. Ao, M. Zheng, J. A. Fagan and A. R. Hight Walker, ACS Nano, 2016, 10, 5252.

40 R. Saito, M. Hofmann, G. Dresselhaus, A. Jorio and M. S. Dresselhaus, Philos. Trans. R. Soc., A, 2008, 366, 231.

41 R. B. Capaz, C. D. Spataru, S. Ismail-Beigi and S. G. Louie, Phys. Status Solidi, 2010, 244, 4016.

42 S. Debnath, Q. Cheng, T. G. Hedderman and H. J. Byrne, Carbon, 2010, 48, 1489.

43 R. B. Weisman and S. M. Bachilo, Nano Lett., 2003, 3, 1235. 44 M. Baibarac, A. Matea, M. Daescu, I. Mercioniu, S. Quillard, J. Y. Mevellec and S. Lefrant, Sci. Rep., 2018, 8, 9518.

45 S. Lebedkin, I. Kareev, F. Hennrich and M. M. Kappes, J. Phys. Chem. C, 2015, 112, 16236.

46 B. Pan, D. Cui, C. S. Ozkan, M. Ozkan, X. Ping, T. Huang, F. Liu, C. Hao, Q. Li and H. Rong, J. Phys. Chem. C, 2008, 112, 939.

47 N. Almeria and R. Paul, ChemInform, 2003, 34, 4139.

48 L. Zhenggang, F. Eduardo and T. Walter, J. Phys. Chem. B, 2009, 113, 3548.

49 J. Yang, D. Zhang, Y. Hu, C. Xia, S. Sun and Y. Li, ACS Nano, 2017, 11, 10509.

50 K. Sato, R. Saito, J. Jiang, G. Dresselhaus and M. S. Dresselhaus, Phys. Rev. B: Condens. Matter Mater. Phys., 2007, 76, 195446.

51 M. P. Scannell, G. Prakash and D. E. Falvey, J. Phys. Chem. A, 1997, 101, 4332.

52 D. T. Lee, J. W. Chung, G. Park, Y. T. Kim, C. Y. Lee, Y. Cho, P. J. Yoo, J. H. Han, H. J. Shin and W. J. Kim, Appl. Surf. Sci., 2018, 429, 264.

53 S. Sayyare and M. Xirali, J. Mater. Res., 2014, 29, 10. 